B. László and J. T. Tóth, Department of Mathematics, University of Education, Farská 19, 94974 Nitra, Slovakia, e-mail: toth@@unitra.sk

\title{
RELATIVELY $(R)$-DENSE UNIVERSAL SEQUENCES FOR CERTAIN CLASSES OF FUNCTIONS
}

\begin{abstract}
Let $f: \mathbb{R}^{+} \rightarrow \mathbb{R}^{+}$and $\left(a_{n}\right)_{n=1}^{\infty}$ be a sequence of positive reals. We will say that $\left(a_{n}\right)_{n=1}^{\infty}$ is relatively $(R)$-dense for $f$ provided that for every $x, y \in \mathbb{R}^{+}$with $f(x)<f(y)$ there exists $n, m \in \mathbb{N}$ such that $f(x)<\frac{f\left(a_{n}\right)}{f\left(a_{m}\right)}<f(y)$. Sufficient conditions are given for a sequence of positive reals to be relatively $(R)$-dense for certain functions.
\end{abstract}

\section{Introduction}

Denote by $\mathbb{R}^{+}$and $\mathbb{N}$ the set of all positive real numbers and the natural numbers, respectively. Let $R(A, B)=\left\{\frac{a}{b} ; a \in A, b \in B\right\}$ be the ratio set of $A, B \subset \mathbb{R}^{+}$and put $R(A)=R(A, A)$ for any $A \subset \mathbb{R}^{+}$(cf. [2], [3], [4], [5]). Note here that $R(A, B) \neq R(B, A)$ in general, however $R(A, B)$ is dense in $\mathbb{R}^{+}$if and only if $R(B, A)$ is dense in $\mathbb{R}^{+}$.

Following [2] and [4] we call a set $A=\left\{a_{1}, a_{2}, \ldots, a_{n}, \ldots\right\} \subset \mathbb{R}^{+}(R)$ dense, provided $R(A)$ is dense in $\mathbb{R}^{+}$. Occasionally we will work with a sequence $A=\left(a_{n}\right)_{n=1}^{\infty}$ rather than a set $A$. Sequences of real numbers that are relatively dense for a function $f$ were introduced and investigated in [1]. A straightforward analogue for $(R)$-density is as follows: Let $f: \mathbb{R}^{+} \rightarrow \mathbb{R}^{+}$and $\left(a_{n}\right)_{n=1}^{\infty}$ be a sequence of positive reals. We will say that $\left(a_{n}\right)_{n=1}^{\infty}$ is relatively $(R)$-dense for $f$ provided that for every $x, y \in \mathbb{R}^{+}$with $f(x)<f(y)$ there

Key Words: sequences, ratio set, (R)-density

Mathematical Reviews subject classification: Primary: 26A99. Secondary: 11B83

Received by the editors Feb 20, 1995

* This research was supported by the Slovak Academy of Sciences Grant 1227 
exists $n, m \in \mathbb{N}$ such that $f(x)<\frac{f\left(a_{n}\right)}{f\left(a_{m}\right)}<f(y)$ (the choice $f(x)=x$ clearly yields the $(R)$-density). Evidently, $\left(a_{n}\right)_{n=1}^{\infty}$ is relatively $(R)$-dense for $f$, if the sequence $f(A)=\left(f\left(a_{n}\right)\right)_{n=1}^{\infty}$ is $(R)$-dense. Further $\left(a_{n}\right)_{n=1}^{\infty}$ will be called a relatively $(R)$-dense universal sequence for the class of functions $\mathcal{M}$, if $\left(a_{n}\right)_{n=1}^{\infty}$ is relatively $(R)$-dense for every $f \in \mathcal{M}$. In what follows $A(x)$ will stand for the counting function of the set $A=\left\{a_{1}<a_{2}<\cdots<a_{n}<\ldots\right\} \subset \mathbb{R}^{+}$with $\lim _{n \rightarrow \infty} a_{n}=+\infty$, i.e. $A(x)=\sum_{a \leq x, a \in A} 1$.

It is the purpose of this paper to study relatively $(R)$-dense universal sequences for a certain class of increasing functions, thus extending several results of [1], [2], [3], [4] concerning sets $R(A, B)$.

\section{Main Results}

Denote by $\mathcal{F}$ the set of all functions $f: \mathbb{R}^{+} \rightarrow \mathbb{R}^{+}$satisfying the following properties:

1. $f(x \cdot y) \geq f(x) \cdot f(y)$ for all $x, y \in \mathbb{R}^{+}$,

2. $f$ is increasing and unbounded on $\mathbb{R}^{+}$,

3. $f$ is continuous at $x=1$ and $f(1)=1$.

We have

Theorem 1 Suppose that the sequence $\left(a_{n}\right)_{n=1}^{\infty}$ of positive reals contains an unbounded subsequence $\left(c_{n}\right)_{n=1}^{\infty}$ such that

$$
\limsup _{n \rightarrow \infty} \frac{c_{n+1}}{c_{n}}=1
$$

Then $\left(a_{n}\right)_{n=1}^{\infty}$ is a relatively $(R)$-dense universal sequence for $\mathcal{F}$.

This theorem is a consequence of the following stronger statement:

Theorem 2 Suppose that $A=\left(a_{n}\right)_{n=1}^{\infty}$ fulfills the conditions of Theorem 1 and $B=\left(b_{n}\right)_{n=1}^{\infty}$ is an unbounded sequence of positive reals. Suppose that $\lim _{x \rightarrow \infty} g(x)=+\infty$ where $g: \mathbb{R}^{+} \rightarrow \mathbb{R}^{+}$. Then $R(f(A), g(B))$ is dense in $\mathbb{R}^{+}$for each $f \in \mathcal{F}$.

Proof. According to (1) $\liminf _{n \rightarrow \infty} \frac{c_{n}}{c_{n+1}}=1$ thus by properties of $\mathcal{F}$ $\liminf _{n \rightarrow \infty} f\left(\frac{c_{n}}{c_{n+1}}\right)=1$, so $\limsup _{n \rightarrow \infty} \frac{n \rightarrow \infty}{f\left(\frac{c_{n}}{c_{n+1}}\right)}=1$. Let $0<a<b$. Then

$$
\frac{1}{f\left(\frac{c_{n}}{c_{n+1}}\right)}<\frac{b}{a}
$$


whenever $n \geq n_{0}$ for some $n_{0} \in \mathbb{N}$. Consequently by the first property of $f$

$$
\frac{f\left(c_{n+1}\right)}{f\left(c_{n}\right)}<\frac{b}{a} \text { for all } n \geq n_{0}
$$

The sequences $\left(b_{n}\right)_{n=1}^{\infty}$ and $\left(c_{n}\right)_{n=1}^{\infty}$ are unbounded so there exists an $i \in \mathbb{N}$ with $f\left(c_{n_{o}}\right) b \leq g\left(b_{i}\right)$ further the number $j=\min \left\{k \in \mathbb{N} ; k>n_{0}\right.$ and $f\left(c_{k}\right) b>$ $\left.g\left(b_{i}\right)\right\}$. Then $j-1 \geq n_{0}$ and $f\left(c_{j}\right) b>g\left(b_{i}\right) \geq f\left(c_{j-1}\right) \cdot b$. Hence from (2) for $n=j-1$ we get that $f\left(c_{j-1}\right) b>f\left(c_{j}\right) a$, so by the previous inequalities $f\left(c_{j}\right) b>g\left(b_{i}\right)>f\left(c_{j}\right) a$ which yields the density of $R(g(B), f(A))$ thus also of $R(f(A), g(B))$ in $\mathbb{R}^{+}$since $c_{j} \in A$.

Remark 1 The preceding theorem generalizes Theorem 2.1 in [1] stating that $R(A, B)$ is dense in $\mathbb{R}^{+}$for every couple of unbounded sequences $A, B$ of positive reals such that $\limsup _{n \rightarrow \infty} \frac{a_{n+1}}{a_{n}}=1$.

Proof of Theorem 1. Let $f \in \mathcal{F}$. It suffices to choose $b_{n}=a_{n}(n=1,2, \ldots)$ and $g(x)=f(x)$ in Theorem 2 .

Remark 2 Let $0<c \leq 1$ and $\alpha \in \mathbb{R} \backslash\{0\}$. The function $f(x)=c x^{\alpha}, x \in \mathbb{R}^{+}$ belongs to $\mathcal{F}$. Further $\mathcal{F}$ also contains functions which are not continuous or strictly increasing, respectively on $\mathbb{R}^{+}$; e.g.

$$
f(x)= \begin{cases}c, & \text { if } x \in\left[1, x_{0}\right) \\ c x^{\alpha}, & \text { if } x \in \mathbb{R}^{+} \backslash\left[1, x_{0}\right) \text { where } x_{0}>1 .\end{cases}
$$

It is proved in [4] (Satz 5) that if $A=\left\{a_{1}<a_{2}<\cdots<a_{n}<\ldots\right\}$, $B=\left\{b_{1}<b_{2}<\cdots<b_{n}<\ldots\right\} \subset \mathbb{N}$ and at least one of $A, B$ has positive asymptotic density (i.e. $\lim _{n \rightarrow \infty} \frac{A(n)}{n}>0$ or $\lim _{n \rightarrow \infty} \frac{B(n)}{n}>0$ ) then $R(A, B)$ is dense in $\mathbb{R}^{+}$. The pertinent theorem for one set is proved in (3, Theorem 4). The following theorem generalizes these results for positive real numbers. Before stating it introduce the class $\mathcal{F}^{*}$ of all functions $f: \mathbb{R}^{+} \rightarrow \mathbb{R}^{+}$for which:

1. $f(x \cdot y) \geq f(x) \cdot f(y)$ for all $x, y \in \mathbb{R}^{+}$,

2. $f(x)>1$ for all $x>1$.

It is not hard to prove that members of $\mathcal{F}^{*}$ are increasing and unbounded on $\mathbb{R}^{+}$, so $\mathcal{F} \not \subset \mathcal{F}^{*}$ and $\mathcal{F}^{*} \not \subset \mathcal{F}$ but $\mathcal{F} \cap \mathcal{F}^{*}$ is an infinite set. We have

Theorem 3 Suppose that $B=\left\{b_{1}, b_{2}, \ldots, b_{n}, \ldots\right\} \subset \mathbb{R}^{+}$is unbounded as well as the set $A=\left\{a_{1}<a_{2}<\cdots<a_{n}<\ldots\right\} \subset \mathbb{R}^{+}$. Suppose that

$$
\lim _{x \rightarrow \infty} \frac{f(A)(x)}{g(x)}>0
$$


for some $g \in \mathcal{F}^{*}$ and a strictly increasing unbounded function $f: \mathbb{R}^{+} \rightarrow \mathbb{R}^{+}$. Then $R(f(A), g(B))$ is dense in $\mathbb{R}^{+}$.

Proof. Let $0<a<b$ and $x \in \mathbb{R}^{+}$. Then by the 1 . property of $g \in \mathcal{F}^{*}$ we get:

$$
\frac{f(A)(b x)}{f(A)(a x)}=\frac{\frac{f(A)(b x)}{g(b x)}}{\frac{f(A)(a x)}{g(a x)}} \cdot \frac{g(b x)}{g(a x)} \geq \frac{\frac{f(A)(b x)}{g(b x)}}{\frac{f(A)(a x)}{g(a x)}} \cdot g\left(\frac{b}{a}\right) .
$$

Then by (3) and the 2nd property of $g, \lim _{x \rightarrow \infty} \frac{f(A)(b x)}{f(A)(a x)} \geq g\left(\frac{b}{a}\right)>1$. Accordingly $\frac{f(A)(b x)}{f(A)(a x)}>1$ whenever $x \geq x_{0}$ for some $x_{0} \in \mathbb{R}^{+}$, hence $f(A)(b x)-f(A)(a x)>$ 0 thus for all $x \geq x_{0}$ there exists $i=i(x) \in \mathbb{N}$ such that $a x<f\left(a_{i}\right)<b x$.

Now $\lim _{x \rightarrow \infty} g(x)=+\infty$ and $B$ is unbounded so $g\left(b_{j}\right)>x_{0}$ for some $j \in \mathbb{N}$. It means by previous considerations that $g\left(b_{j}\right) a<f\left(a_{i}\right)<g\left(b_{j}\right) b$ for some $i=i\left(g\left(b_{j}\right)\right) \in \mathbb{N}$ and the density of $R(f(A), g(B))$ in $\mathbb{R}^{+}$follows.

If $X^{d}$ denotes the set of all limit points of the set $X$ then we have:

Theorem 4 If $A=\left\{a_{1}<a_{2}<\ldots a_{n}<\ldots\right\} \subset \mathbb{R}^{+}$is relatively $(R)$-dense for some $f \in \mathcal{F}^{*}$, then $\liminf _{n \rightarrow \infty} \frac{a_{n+1}}{a_{n}}=1$.

Proof. Let $f \in \mathcal{F}^{*}$ and suppose that $\liminf _{n \rightarrow \infty} \frac{a_{n+1}}{a_{n}}=c>1$, where $c \in \mathbb{R}^{+}$. It follows from properties of $f$ that

$$
\liminf _{n \rightarrow \infty} f\left(\frac{a_{n+1}}{a_{n}}\right) \geq f(c)>1 \text { and } \frac{f\left(a_{n+1}\right)}{f\left(a_{n}\right)} \geq f\left(\frac{a_{n+1}}{a_{n}}\right),
$$

thus $\liminf _{n \rightarrow \infty} \frac{f\left(a_{n+1}\right)}{f\left(a_{n}\right)} \geq f(c)$. If $c=+\infty$ then put $f(c)=+\infty$.

Now choose $t \in R(f(A))^{d}$ and $t>1$. Then $\frac{f\left(a_{m_{k}}\right)}{f\left(a_{n_{k}}\right)} \rightarrow t($ as $k \rightarrow \infty)$ for some sequences $\left(m_{k}\right)_{k=1}^{\infty},\left(n_{k}\right)_{k=1}^{\infty}$ of natural numbers such that $m_{k}>n_{k}$ $(k=1,2, \ldots)$. Clearly $\frac{f\left(a_{m_{k}}\right)}{f\left(a_{n_{k}}\right)} \geq \frac{f\left(a_{\left.n_{k}+1\right)}\right)}{f\left(a_{n_{k}}\right)}$ so

$$
t \geq \liminf _{k \rightarrow \infty} \frac{f\left(a_{n_{k+1}}\right)}{f\left(a_{n_{k}}\right)} \geq \liminf _{n \rightarrow \infty} \frac{f\left(a_{n+1}\right)}{f\left(a_{n}\right)} \geq f(c) .
$$

Consequently $R(f(A))^{d} \cap(1, f(c))=\emptyset$. Then properties of $f$ easily yield numbers $1<x<y<c$ such that $1<f(x)<f(y)<f(c)$ and $(f(x), f(y)) \cap$ $R(f(A))=\emptyset$, whence $A$ is not relatively $(R)$-dense for $f$.

Remark 3 Functions $f(x)=x^{\alpha}, x \in \mathbb{R}^{+}, \alpha \in \mathbb{R} \backslash\{0\}$ belong to $\mathcal{F} \cap \mathcal{F}^{*}$, so these functions satisfy Theorem 1 and Theorem 4. 
An argument similar to that of in Theorem 4 justifies that $t \leq \frac{1}{f(c)}$ if $t \in R(f(A))^{d}$ and $t<1$. Hence we have the following generalization of Proposition 3 of $[6]$ :

Corollary 1 Let $f \in \mathcal{F}^{*}$ and $A=\left\{a_{1}<a_{2}<\ldots a_{n}<\ldots\right\} \subset \mathbb{R}^{+}$such that $\liminf _{n \rightarrow \infty} \frac{a_{n+1}}{a_{n}}=c>1$. Then $R(f(A))^{d} \cap\left(\frac{1}{f(c)}, f(c)\right)=\emptyset$.

Remark 4 If $c=+\infty$ in the preceding Corollary, then $R(f(A))$ consists of isolated points in $\mathbb{R}^{+}$.

Remark 5 It was already mentioned in the introduction that $(R)$-density of $f(A)$ implies relative $(R)$-density of $A=\left(a_{n}\right)_{n=1}^{\infty}$ for a function $f$. It is evident that the reverse implication also holds for surjective $f: \mathbb{R}^{+} \rightarrow \mathbb{R}^{+}$.

In connection with this it would be interesting to characterize functions $f: \mathbb{R}^{+} \rightarrow \mathbb{R}^{+}$for which $f(A)$ is $(R)$-dense if and only if the sequence $A=$ $\left(a_{n}\right)_{n=1}^{\infty}$ is relatively $(R)$-dense for $f$.

\section{References}

[1] D. Andrica and S. Buzeteanu, Relatively dense universal sequences for the class of continuous periodical functions of period T, L' Analyse Numérique et la théorie de L'Approximation, 16 no. 1 (1987), 1-9.

[2] W. Narkiewicz and T. Šalát, A theorem of H. Steinhaus and $(R)$-dense sets of positive integers, Czechoslov. Math. J., 34 (109) (1984), 355-361.

[3] T. Šalát, On ratio sets of sets of natural numbers, Acta Arith., 15 (1969), $273-278$.

[4] T. Šalát, Quotientbasen und (R)-dichte Mengen, Acta Arith., 19 (1971), 63-78.

[5] J. Smítal, Remarks on ratio sets of natural numbers, Acta F. R. N. Univ. Comen. - Math., 25 (1971), 93-99.

[6] J. T. Tóth and L. Zsilinszky, On density of ratio sets of powers of primes, Nieuw Archief voor Wiskunde, 13 no. 2 (1995), 205-208. 